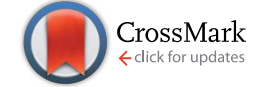

Cite this: RSC Adv., 2016, 6, 10054

Received 8th October 2015

Accepted 15th January 2016

DOI: $10.1039 / \mathrm{c} 5 \mathrm{ra20848c}$

www.rsc.org/advances

\section{Synthesis and characterization of functionalized molecular and macromolecular double-decker silsesquioxane systems $\uparrow$}

\author{
P. Żak, ${ }^{a}$ M. Majchrzak, ${ }^{a}$ G. Wilkowski, ${ }^{a}$ B. Dudziec, ${ }^{a}$ M. Dutkiewicz ${ }^{b}$ and B. Marciniec ${ }^{\star b}$ \\ Synthesis and characterization of new, molecular DDSQ-based compounds functionalized by substituted \\ styrenes (also with heteroatoms) in a stereoselective manner is presented. Additionally, a novel class of \\ vinylene-arylene linear copolymers with double-decker silsesquioxane fragments in the main chain is \\ developed. These molecular and macromolecular compounds were synthesized via efficient and highly \\ stereoselective silylative coupling (and copolycondensation) and cross-metathesis (and ADMET) reactions.
}

\section{Introduction}

Polyhedral oligomeric silsesquioxanes (POSS) of the empirical formula $\left(\mathrm{RSiO}_{1.5}\right)_{n}$ with different reactive and/or inert organic coronae, have recently become the subject of growing interest in many branches of chemistry. ${ }^{1}$ It is known that cage structure silsesquioxanes, due to their unique properties, e.g. the rigid inorganic SiO core with a diameter of about $1.5 \mathrm{~nm}$, are often regarded as the smallest silica particles that can also influence the surface properties of functional groups attached to silicon atoms. A well-defined organization of POSS is important because of their possible use as precursors and components of versatile inorganic/organic hybrid materials that display nanophase behavior. Not more than a decade ago, a new class of silsesquioxyl compounds containing rigid $\mathrm{Si}-\mathrm{O}-\mathrm{Si}$ bonds, i.e. the double-decker silsesquioxane (DDSQ), was reported by Yoshida and co-workers. ${ }^{2}$ The structure of DDSQ is an open cage that includes two cyclosiloxane rings in parallel planes with 8 inert phenyl groups at the silicon atoms of each ring. These two rings are joined by oxygen bridges. Current research on that compound refers to two major trends in its development and knowledge, i.e. syntheses of molecular and macromolecular organosilicon compounds based on DDSQ that may exhibit interesting and desired physicochemical properties. ${ }^{3}$

The silylative coupling ( $\mathbf{S C}$ ) and cross-metathesis (CM) of olefins with vinyl-substituted organosilicon compounds have been studied and developed for the last two decades as a new, effective catalytic activation of the $=\mathrm{C}-\mathrm{H}$ bond of olefins and the $=\mathrm{C}-\mathrm{Si}$ bond of organosilicon compounds (Scheme 1). The

${ }^{a}$ Department of Organometallic Chemistry, Faculty of Chemistry, Adam Mickiewicz University in Poznan, Umultowska 89B, 61-614 Poznan, Poland

${ }^{b}$ Centre for Advanced Technologies, Adam Mickiewicz University in Poznan, Umultowska 89C, 61-614 Poznan, Poland. E-mail: Bogdan.Marciniec@amu.edu.pl

$\uparrow$ Electronic supplementary information (ESI) available. See DOI: $10.1039 / \mathrm{c} 5 \mathrm{ra} 20848 \mathrm{c}$ mechanisms of these reactions are diverse as they require different catalysts (mostly ruthenium hydride for SC and ruthenium-carbene for $\mathbf{C M}$ ). However, both $\mathbf{S C}$ and $\mathbf{C M}$ proved to be effective paths for synthesis of functional vinylsilicon molecular as well as macromolecular compounds. ${ }^{4}$

In the previous decade, we reported both processes (SC and CM) to be complementary routes for regio- and stereoselective synthesis of functionalized vinylsilsesquioxanes and vinylspherosilicates. ${ }^{5}$ Cross-metathesis of vinylsilsesquioxanes using Grubbs catalyst was exploited by Feher, ${ }^{6}$ Laine, ${ }^{7}$ Cole-Hamilton and co-workers ${ }^{8}$ as well as Naka. ${ }^{9}$ Schrock noted the use of molybdenum catalyst for $\mathbf{C M}$ but in all reactions, except for styrene, a mixture of stereoisomers was obtained. ${ }^{6}$ On the other hand, hydrosilylation and Heck reaction were applied for vinylsubstituted POSS modification to obtain compounds of interesting photophysical properties enabling their use as sugars and peptide scaffolds. ${ }^{8 a, 10}$

Our recent report has proved a successful use of divinylsubstituted double-decker silsesquioxanes (DDSQ-2SiVi 1A and

Silylative Coupling (SC)

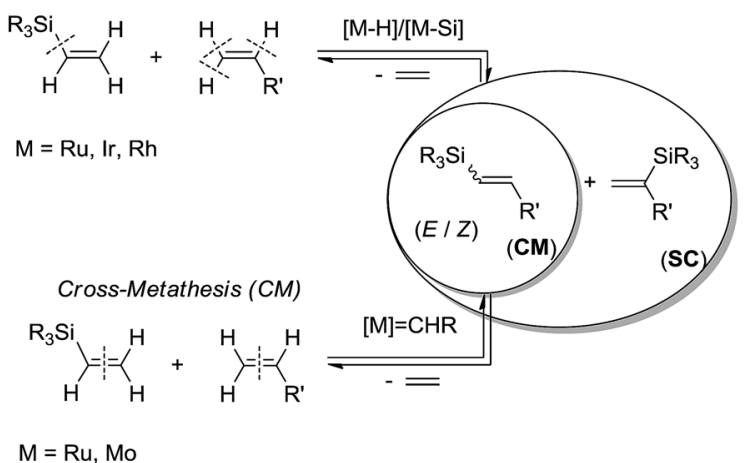

Scheme 1 Difference in regioselectivity of silylative coupling (SC) and cross-metathesis $(\mathrm{CM})$ reactions of vinylsilanes with olefins. 


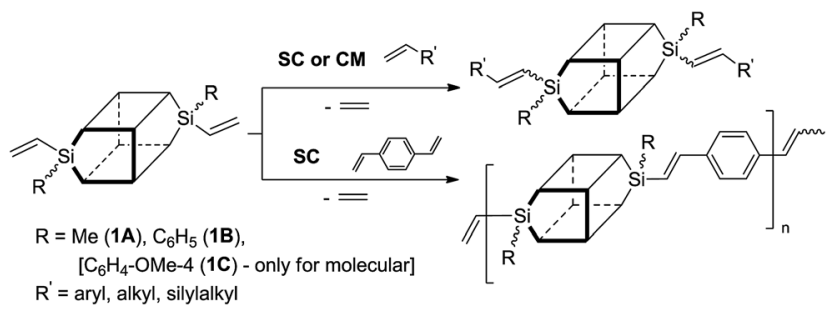

Scheme 2 Protocol using DDSQ-2SiVi for stereoselective silylative coupling (also copolycondensation) and cross-metathesis with olefins.

1B) as substrates for highly effective silylative coupling (SC) and/ or cross-metathesis (CM) with olefins (Scheme 2). ${ }^{11}$ The reactions led to highly stereoselective formation of $E$ isomers. Preliminary studies on silylative coupling copolycondensation (SCC) of divinylbenzene (DVB) with DDSQ-2SiVi show a possibility to obtain stereoregular, linear cooligomer containing DDSQ-vinylene-phenylene units in the chain.

Here, we present a synthetic path for obtaining new, molecular DDSQ-based compounds functionalized by substituted styrenes (also with heretoatoms) in a stereoselective manner as well as a new class of vinylene-arylene copolymers with double-decker silsesquioxane in the main chain. All of the compounds were characterized via spectroscopic methods and their structural properties were also studied.

\section{Experimental}

\section{Materials and methods}

All reactions were carried out under argon atmosphere using standard Schlenk-line and vacuum techniques. All solvents were dried prior to use over $\mathrm{CaH}_{2}$ and stored under argon over molecular sieves type 4 A. Dichloromethane was additionally passed through a column with alumina and after that it was degassed by repeated freeze-pump-thaw cycles.

The chemicals were purchased from the following sources: dichloromethane (DCM), tetrahydrofuran (THF), $n$-hexane, chloroform-d, dichloromethane- $\mathrm{d}_{2}$, ethanol, methanol, acetone, calcium hydride, diethyl ether, tricyclohexylphosphine, 9-bromoanthracene, 1-bromonaphthalene, 2-bromothiophene, 2,5dibromothiophene, bromopentafluorobenzen, 4-vinylphenylboronic acid, 1,4-dibromo-2,3,5,6-tetrafluorobenzene, 9,10-dibromoanthracene, 1,4-dibromonaphthalene, molecular sieves type $4 \AA$, copper(I) chloride and anhydrous magnesium sulfate from Aldrich, vinylmethyldichlorosilane and vinylphenyldichlorosilane from ABCR, Grubbs first generation benzylidene catalyst from Apeiron Synthesis, triethylamine and silica gel 60 from Fluka, ruthenium(III) chloride hydrate from Lancaster, tetrasilanolphenyl POSS $\left(\mathrm{Ph}_{8} \mathrm{O}_{8} \mathrm{Si}_{8}\right)(\mathrm{OH})_{4}(\mathbf{D D S Q}-4 \mathrm{OH})$ from Hybrid Plastics. Divinyl-substituted double-decker silsesquioxanes (DDSQ-2SiVi) (1A and 1B) were prepared according to the literature procedure. ${ }^{11 b}$ All mono- and distyrylarenes were synthesized according to the literature procedure. ${ }^{12}$ The ruthenium species $\left[\mathrm{RuHCl}(\mathrm{CO})\left(\mathrm{PCy}_{3}\right)_{2}\right]$ was made in accordance with the procedure described in literature. ${ }^{13}$

\section{Techniques and measurement}

Nuclear magnetic resonance (NMR) spectroscopy. NMR spectra were recorded at $25{ }^{\circ} \mathrm{C}$ on a Brucker Ultra Shield (300 and $400 \mathrm{MHz}$ ) spectrometers, $\mathrm{CDCl}_{3}, \mathrm{CD}_{2} \mathrm{Cl}_{2}$ were used as solvents and for internal deuterium lock. Chemical shifts are reported in $\mathrm{ppm}$ with reference to the residual solvents $\left(\mathrm{CHCl}_{3}, \mathrm{CH}_{2} \mathrm{Cl}_{2}\right)$ peaks for ${ }^{1} \mathrm{H}$ and ${ }^{13} \mathrm{C}$ NMR and to TMS for ${ }^{29} \mathrm{Si}$ NMR.

Thin layer chromatography (TLC). TLC was conducted on plates coated with $250 \mathrm{~mm}$ thick silica gel and column chromatography was performed on silica gel 60 (70-230 mesh) using a mixture of $n$-hexane/DCM.

Gel permeation chromatography (GPC). GPC analyses were performed using a Waters 2414 RI detector and a set of three serially connected $7.8 \times 300 \mathrm{~mm}$ columns (Waters Styragel HR1, HR2 and HR4). THF was used as a mobile phase at the flow rate of $0.6 \mathrm{~mL} \mathrm{~min}^{-1}$; column oven temperature was $35{ }^{\circ} \mathrm{C}$ and detector temperature $40{ }^{\circ} \mathrm{C}$. All molecular weight $\left(M_{\mathrm{n}}, M_{\mathrm{w}}\right)$ and polydispersity index (PDI) values were calculated on the basis of the calibration curve using polystyrene standards (Shodex) in the range from $1.31 \times 10^{3}$ to $3.64 \times 10^{6} \mathrm{Da}$.

Thermal gravimetric analysis (TGA). A TA Instruments TGA Q50 analyzer was used to investigate thermal stability of the samples. The measurements were conducted in nitrogen flow of $60 \mathrm{~mL} \mathrm{~min}{ }^{-1}$ from ambient temperature to $1000{ }^{\circ} \mathrm{C}$ at the heating rate of $10{ }^{\circ} \mathrm{C} \mathrm{min}^{-1}$. The temperature of initial degradation $\left(T_{\mathrm{d}}\right)$ was taken as the onset temperature of initial degradation $\left(T_{\mathrm{d}}\right)$ at which $5 \mathrm{wt} \%$ of mass loss occurs.

\section{General procedure for the synthesis of functionalized $E, E$ - vinylene-arylenesilsesquioxanes (1-8)}

By cross-metathesis (CM) with olefins. An oven-dried $5 \mathrm{~mL}$ glass reactor equipped with a reflux condenser and magnetic stirring bar was charged under argon with divinylsilsesquioxane (1B) $\left(0.1 \mathrm{~g}, 7.52 \times 10^{-5} \mathrm{~mol}\right)$, olefin $\left(1.50 \times 10^{-4} \mathrm{~mol}\right)$ and dichloromethane $(2 \mathrm{~mL})$. The reaction mixture was stirred and heated in an oil bath to maintain a gentle reflux (ca. $\left.45{ }^{\circ} \mathrm{C}\right)$. Then the first generation Grubbs' catalyst $\left(0.0012 \mathrm{~g}, 1.50 \times 10^{-6}\right.$ mol) was added to the mixture under argon. The reaction mixture was heated under reflux for $24 \mathrm{~h}$. Then the solvent was evaporated under a vacuum and cold $n$-hexane $(2 \mathrm{~mL})$ was added to the remaining content to form precipitate. The precipitate was filtered off and purified by column chromatography (silica gel 60/n-hexane : $\mathrm{CH}_{2} \mathrm{Cl}_{2}=1: 5$ ) to remove ruthenium complexes. Evaporation of the solvent gave an analytically pure sample (white or yellow powder).

By silylative coupling (SC) with olefins. For the syntheses of molecular compounds the complex ruthenium(II)-RuHCl(CO)$\left(\mathrm{PCy}_{3}\right)_{2}$ was used as a catalyst. Toluene and dichloromethane were dried and deoxygenated and all operations were performed under argon.

System 1. A $5 \mathrm{~mL}$ two-necked glass reactor equipped with a reflux condenser and connected to a bubbler was charged under argon with divinylsilsesquioxane (1A) $\left(0.05 \mathrm{~g}, 4.13 \times 10^{-5}\right.$ 
$\mathrm{mol})$, toluene $(1 \mathrm{~mL})$ and styrylarylene derivative $\left(1.24 \times 10^{-4}\right.$ mol). The reaction mixture was heated up to $90{ }^{\circ} \mathrm{C}$ (or $110{ }^{\circ} \mathrm{C}$ for thiophene derivatives) and $\left[\mathrm{RuHCl}(\mathrm{CO})\left(\mathrm{PCy}_{3}\right)_{2}\right](0.0006 \mathrm{~g}$, $8.26 \times 10^{-7} \mathrm{~mol}$ ) was added and the catalytic system was left for $24 \mathrm{~h}$ (except thiophene derivative for $96 \mathrm{~h}$ ). Then the solvent was evaporated under vacuum and the crude product was dissolved in DCM $(1 \mathrm{~mL})$. After that, the brown solution was filtered at 'flash-system' (glass filter-SiO ${ }_{2}-$ Celite) using $n$-hexane three times $(3 \times 10 \mathrm{~mL})$ to remove the excess of olefin and the final product was washed by DCM $(2 \times 10 \mathrm{~mL})$. Evaporation of the solvent gave an analytically pure sample (white or yellow powder).

System 2. A $5 \mathrm{~mL}$ two-necked glass reactor equipped with a reflux condenser and connected to a bubbler was charged under argon with divinylsilsesquioxane (1A) (0.05 g, $4.13 \times$ $\left.10^{-5} \mathrm{~mol}\right)$, dichloromethane $(2 \mathrm{~mL})$ and styrylarylene derivative $\left(8.26 \times 10^{-5} \mathrm{~mol}\right)$. The reaction mixture was stirred and heated in an oil bath to maintain a gentle reflux $\left(\mathrm{ca} .45^{\circ} \mathrm{C}\right)$. Then the hydride ruthenium complex $\left[\mathrm{RuHCl}(\mathrm{CO})\left(\mathrm{PCy}_{3}\right)_{2}\right]$ $\left(0.0006 \mathrm{~g}, 8.26 \times 10^{-7} \mathrm{~mol}\right)$ was added to the mixture under argon. After 5 min copper(I) chloride $\left(0.0004 \mathrm{~g}, 4.13 \times 10^{-6}\right.$ mol) was added. The reaction mixture was heated under reflux for $24 \mathrm{~h}$. Then the solvent was evaporated under vacuum and cold $n$-hexane $(2 \mathrm{~mL})$ was added to the remaining content to form precipitate. The precipitate was filtered off and purified by column chromatography (silica gel 60/n-hexane : $\mathrm{CH}_{2} \mathrm{Cl}_{2}=$ $1: 5)$ to remove ruthenium complexes. Evaporation of the solvent gave an analytically pure sample (white or yellow powder).

\section{Analytical data for isolated molecular compounds (1-8)}

1: trans di[9,19-(E)-4-(9-anthracenyl)styrylphenyl]-1,3,5,7,11, 13,15,17-octaphenylpentacyclo[11.7.1.1 $\left.1^{3,11} \cdot 1^{5,17} \cdot 1^{7,15}\right]$ decasiloxane. ${ }^{1} \mathrm{H} \mathrm{NMR}\left(400 \mathrm{MHz}, \mathrm{CD}_{2} \mathrm{Cl}_{2}, \delta, \mathrm{ppm}\right): 6.85\left(\mathrm{~d}, J_{\mathrm{HH}}=19.2 \mathrm{~Hz}\right.$, $2 \mathrm{H},=\mathrm{CH}-\mathrm{Si}), 7.05-8.61\left(\mathrm{~m}, 78 \mathrm{H},=\mathrm{CH}-\mathrm{C}_{6} \mathrm{H}_{4}-\right.$ and aromatic parts); ${ }^{13} \mathrm{C}$ NMR (125 MHz, $\left.\mathrm{CD}_{2} \mathrm{Cl}_{2}, \delta, \mathrm{ppm}\right): 123.36,125.19$, 125.45 , 126.62, 126.97, 126.83, 127.56, 127.60, 127.65, 127.71, 127.77, 127.94, 127.99, 128.47, 129.61, 130.44 (d, $J=4.4 \mathrm{~Hz})$, $130.71,131.51,134.00,134.05,134.11,134.16,137.38,140.12$, 147.53, 165.39; ${ }^{29} \mathrm{Si} \mathrm{NMR}\left(79 \mathrm{MHz}, \mathrm{CD}_{2} \mathrm{Cl}_{2}, \delta\right.$ (ppm)): -44.36, $-77.75,-79.21$.

2: trans di[9,19-(E)-4-(1-naphthyl)styrylphenyl]-1,3,5,7,11,13, 15,17-octaphenylpentacyclo[11.7.1.1 $\left.{ }^{3,11} \cdot 1^{5,17} \cdot 1^{7,15}\right]$ decasiloxane. ${ }^{1} \mathrm{H}$ NMR (400 MHz, $\mathrm{CD}_{2} \mathrm{Cl}_{2}, \delta, \mathrm{ppm}$ ): 6.75 (overlapping dublets, $J_{\mathrm{HH}}=19.2 \mathrm{~Hz}, 4 \mathrm{H},=\mathrm{CH}-\mathrm{Si}$ and $\left.=\mathrm{CH}-\mathrm{C}_{6} \mathrm{H}_{4}{ }^{-}\right), 7.02-8.28(\mathrm{~m}$, $72 \mathrm{H}$, aromatic parts); ${ }^{13} \mathrm{C} \mathrm{NMR}\left(125 \mathrm{MHz}, \mathrm{CD}_{2} \mathrm{Cl}_{2}, \delta, \mathrm{ppm}\right)$ : 122.31, 125.40, 125.82, 126.07, 126.84 (br s), 127.54, 127.61, 127.67, 127.70, 127.95, 128.25, 130.19, 130.34, 130.46 (br s), 130.65 , 131.44, 131.56, 133.85, 133.99, 134.02, 134.06, 134.08, $134.15,134.50,136.42,139.75,141.11 ;{ }^{29} \mathrm{Si}$ NMR (79 MHz, $\left.\mathrm{CD}_{2} \mathrm{Cl}_{2}, \delta, \mathrm{ppm}\right):-44.37,-77.83,-79.31$.

3: trans di[9,19-(E)-4-(2,3,4,5,6-pentafluorophenyl)styryl-phenyl]-

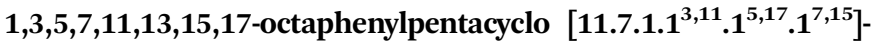
decasiloxane. ${ }^{1} \mathrm{H}$ NMR $\left(400 \mathrm{MHz}, \mathrm{CD}_{2} \mathrm{Cl}_{2}, \delta\right.$, ppm): $6.73\left(\mathrm{~d}, J_{\mathrm{HH}}=\right.$ $20.0 \mathrm{~Hz}, 2 \mathrm{H},=\mathrm{CH}-\mathrm{Si}), 6.97-8.15\left(\mathrm{~m}, 60 \mathrm{H},=\mathrm{CH}-\mathrm{C}_{6} \mathrm{~F}_{4}\right.$ and aromatic parts); ${ }^{13} \mathrm{C}$ NMR (125 $\left.\mathrm{MHz}, \mathrm{CD}_{2} \mathrm{Cl}_{2}, \delta, \mathrm{ppm}\right): 121.80$,
124.13, 127.07, $127.59(\mathrm{t}, J=5.9 \mathrm{~Hz}), 127.88,127.95,128.02$, $130.26(\mathrm{t}, J=4.9 \mathrm{~Hz}), 130.36,130.41,130.47,130.67(\mathrm{t}, J=2.4$ $\mathrm{Hz}), 131.43$ ( $\mathrm{t}, J=2.1 \mathrm{~Hz}), 133.94,133.98,134.02,138.32,147.08$; ${ }^{29} \mathrm{Si} \mathrm{NMR}\left(79 \mathrm{MHz}, \mathrm{CD}_{2} \mathrm{Cl}_{2}, \delta, \mathrm{ppm}\right):-44.78,-77.83,-79.33$.

4: trans di[9,19-(E)-4-(2-thienyl)styryl-phenyl]-1,3,5,7,11,13, 15,17octaphenylpentacyclo[11.7.1.1 $\left.1^{3,11} \cdot 1^{5,17} \cdot 1^{7,15}\right]$ decasiloxane. ${ }^{1} \mathrm{H}$ NMR (400 MHz, $\left.\mathrm{CD}_{2} \mathrm{Cl}_{2}, \delta, \mathrm{ppm}\right): 6.65\left(\mathrm{~d}, J_{\mathrm{HH}}=19.2 \mathrm{~Hz}, 2 \mathrm{H}\right.$, $=\mathrm{CH}-\mathrm{Si}), 7.05-8.28\left(\mathrm{~m}, 66 \mathrm{H},=\mathrm{CH}-\mathrm{C}_{6} \mathrm{H}_{4}, \mathrm{C}_{6} \mathrm{H}_{5}, \mathrm{C}_{6} \mathrm{H}_{4}\right.$ and $\mathrm{C}_{4} \mathrm{H}_{3} \mathrm{~S}$ ); ${ }^{13} \mathrm{C}$ NMR (125 MHz, $\left.\mathrm{CD}_{2} \mathrm{Cl}_{2}, \delta, \mathrm{ppm}\right): 122.15,123.32$, 125.12 , 125.75, 127.44, 127.50, 127.59, 127.67, 127.93, 128.15, $129.60,130.33(\mathrm{t}, J=4.3 \mathrm{~Hz}), 130.44(\mathrm{t}, J=3.0 \mathrm{~Hz}), 130.63$, $131.52,134.00(\mathrm{~d}, J=6.1 \mathrm{~Hz}), 134.47,134.51,136.56,143.85 ;{ }^{29} \mathrm{Si}$ NMR (79 MHz, $\left.\mathrm{CD}_{2} \mathrm{Cl}_{2}, \delta, \mathrm{ppm}\right):-44.39,-77.89,-79.34$.

5: cis and trans mixture of $\mathrm{di}[9,19-(E)-4-(9-a n t h r a c e n y l)$ styryl-methyl]-1,3,5,7,11,13,15,17-octaphenylpentacyclo $\quad[11.7$. $\left.\mathbf{1 . 1}^{3,11} .1^{5,17} \cdot 1^{7,15}\right]$ decasiloxane. ${ }^{1} \mathrm{H}$ NMR (300 $\mathrm{MHz}, \mathrm{CDCl}_{3}, \delta$, ppm): 0.49 (overlapping s, $6 \mathrm{H},-\mathrm{SiCH}_{3}$ ), $6.61\left(\mathrm{~d}, 2 \mathrm{H}, J_{\mathrm{HH}}=19.2\right.$ $\mathrm{Hz},=\mathrm{CH}-\mathrm{Si}), 6.95-8.08\left(\mathrm{~m}, 68 \mathrm{H},=\mathrm{CH}-\mathrm{C}_{6} \mathrm{H}_{4}-\right.$ and aromatic parts); $\left.{ }^{13} \mathrm{C} \mathrm{NMR} \mathrm{(75} \mathrm{MHz,} \mathrm{CDCl}_{3}, \delta, \mathrm{ppm}\right):-0.75,-0.65,126.13$, $126.24,127.17,127.30,127.66,127.74,127.80,128.01,130.36$, 130.49, 130.63, 130.88, 131.11, 132.00, 134.11, 134.27, 138.60, $145.63 ;{ }^{29} \mathrm{Si} \mathrm{NMR}\left(79 \mathrm{MHz}, \mathrm{CDCl}_{3}, \delta, \mathrm{ppm}\right):-30.91,-78.18$, $-79.28,-79.46,-79.52$.

6: cis and trans mixture of $\operatorname{di}[9,19-(E)-4-(1-n a p h t h y l)-$ styryl-methyl]-1,3,5,7,11,13,15,17-octaphenylpentacyclo [11.7.1. $\left.\mathbf{1}^{3,11} .1^{5,17} \cdot \mathbf{1}^{7,15}\right]$ decasiloxane. ${ }^{1} \mathrm{H}$ NMR (300 $\mathrm{MHz} \mathrm{CDCl}_{3}, \delta$, ppm): 0.47 (overlapping s, $\left.6 \mathrm{H},-\mathrm{SiCH}_{3}\right), 6.54\left(\mathrm{~d}, 2 \mathrm{H}, J_{\mathrm{HH}}=19.5\right.$ $\mathrm{Hz},=\mathrm{CH}-\mathrm{Si}), 6.88-7.94\left(\mathrm{~m}, 64 \mathrm{H},=\mathrm{CH}-\mathrm{C}_{6} \mathrm{H}_{4}-\right.$ and aromatic parts); ${ }^{13} \mathrm{C}$ NMR (75 MHz, $\left.\mathrm{CDCl}_{3}, \delta, \mathrm{ppm}\right):-0.69,-0.68,125.45$, 125.88, 125.99, 126.09, 126.80, 127.57, 127.64, 127.69, 127.79, $127.91,128.39,130.20,130.36,130.41,130.47,130.92,131.57$, 132.05, 133.86, 134.06, 134.26, 136.62, 139.93, 140.89, 146.29; ${ }^{29} \mathrm{Si} \mathrm{NMR}\left(79 \mathrm{MHz}, \mathrm{CDCl}_{3}, \delta, \mathrm{ppm}\right):-30.13,-78.23,-79.07$, $-79.47,-79.86$.

7: cis and trans mixture of $\operatorname{di}[9,19-(E)-4-(2,3,4,5,6-$ penta-fluorophenyl)styrylmethyl]-1,3,5,7,11,13,15,17-octaphenyl-pentacyclo[11.7.1.1 ${ }^{3,11} \cdot 1^{5,17} \cdot 1^{7,15}$ decasiloxane. ${ }^{1} \mathrm{H}$ NMR $(300 \mathrm{MHz}$, $\left.\mathrm{CDCl}_{3}, \delta, \mathrm{ppm}\right): 0.43\left(\mathrm{~s}, 3 \mathrm{H},-\mathrm{SiCH}_{3}\right), 0.44\left(\mathrm{~s}, 3 \mathrm{H},-\mathrm{SiCH}_{3}\right), 6.51$ $\left(\mathrm{d}, 2 \mathrm{H}, J_{\mathrm{HH}}=18.9 \mathrm{~Hz},=\mathrm{CH}-\mathrm{Si}\right), 6.86-7.61\left(\mathrm{~m}, 50 \mathrm{H},=\mathrm{CH}-\mathrm{C}_{6} \mathrm{~F}_{4}-\right.$ and aromatic parts); $\left.{ }^{13} \mathrm{C} \mathrm{NMR} \mathrm{(75} \mathrm{MHz}, \mathrm{CDCl}_{3}, \delta, \mathrm{ppm}\right):-0.84$, $-0.71,126.13,126.24,127.17,127.30,127.66,127.74,127.93$, $128.01,130.37,130.49,130.57,130.64,130.88,131.11,132.01$, 134.11, 134.27, 138.59, 145.61; ${ }^{29} \mathrm{Si} \mathrm{NMR}\left(79 \mathrm{MHz}, \mathrm{CDC}_{3}, \delta\right.$, ppm): $-30.57,-78.26,-79.18,-79.53,-79.84$.

8: cis and trans mixture of $\operatorname{di}[9,19-(E)-4-(2-$ thienyl)styryl-methyl]-1,3,5,7,11,13,15,17-octaphenylpentacyclo [11.7.1. $\mathbf{1}^{3,11} \cdot \mathbf{1}^{\mathbf{5 , 1 7}} \cdot \mathbf{1}^{\mathbf{7 , 1 5}}$ ]decasiloxane. ${ }^{1} \mathrm{H}$ NMR (300 $\mathrm{MHz}, \mathrm{CDCl}_{3}, \delta$, ppm): 0.49 (overlapping s, $6 \mathrm{H},-\mathrm{SiCH}_{3}$ ), $6.48\left(\mathrm{~d}, 2 \mathrm{H}, J_{\mathrm{HH}}=19.2\right.$ $\mathrm{Hz},=\mathrm{CH}-\mathrm{Si}$ ), 6.98-7.66 (m, 42H, aromatic parts, $2 \mathrm{H}$ coming from $=\mathrm{CH}-\mathrm{C}_{6} \mathrm{H}_{4}-$ and $2 \mathrm{H}$ coming from $\left.-\mathrm{C}_{4} \mathrm{H}_{3} \mathrm{~S}-\right), 7.21(\mathrm{~d}, 2 \mathrm{H}$, $\left.J_{\mathrm{HH}}=6.0 \mathrm{~Hz},-\mathrm{C}_{4} H_{3} \mathrm{~S}-\right), 7.53\left(\mathrm{~d}, 4 \mathrm{H}, J_{\mathrm{HH}}=7.2 \mathrm{~Hz},-\mathrm{C}_{6} H_{4}-\right), 7.70$ $\left(\mathrm{d}, 2 \mathrm{H}, J_{\mathrm{HH}}=9.0 \mathrm{~Hz},-\mathrm{C}_{4} H_{3} \mathrm{~S}-\right), 7.74\left(\mathrm{~d}, 4 \mathrm{H}, J_{\mathrm{HH}}=9.0 \mathrm{~Hz},-\mathrm{C}_{6} H_{4}-\right)$, $7.87\left(\mathrm{~d}, 4 \mathrm{H}, J_{\mathrm{HH}}=7.2 \mathrm{~Hz},-\mathrm{C}_{6} \mathrm{H}_{4}-\right) . ;{ }^{13} \mathrm{C} \mathrm{NMR}\left(75 \mathrm{MHz}, \mathrm{CDCl}_{3}, \delta\right.$, ppm): $-0.62,-0.58,123.30,124.22,125.12,125.98,127.47$, $127.74,127.97,128.24,130.55$, 130.97, 132.10, 134.13, 134.27, 134.46, 136.87, 144.26, 146.07; ${ }^{29} \mathrm{Si} \mathrm{NMR}\left(79 \mathrm{MHz}, \mathrm{CDCl}_{3}, \delta\right.$, ppm): $-30.17,-78.25,-79.20,-79.50,-79.77$. 
General procedure for the synthesis of functionalized $E, E$ arylene-silsesquioxanes derivatives oligomers (9-15)

By metathetic copolymerization (ADMET). A $5 \mathrm{~mL}$ glass reactor equipped with a reflux condenser and magnetic stirring bar was charged under argon with divinylsilsesquioxane (1B) $\left(0.1 \mathrm{~g}, 7.52 \times 10^{-5} \mathrm{~mol}\right)$, diolefin $\left(7.52 \times 10^{-5} \mathrm{~mol}\right)$ and dichloromethane $(2 \mathrm{~mL})$. The reaction mixture was stirred and heated in an oil bath to maintain a gentle reflux (ca. $\left.45{ }^{\circ} \mathrm{C}\right)$. Then, the first generation Grubbs' catalyst $\left(0.0012 \mathrm{~g}, 1.50 \times 10^{-6}\right.$ mol) was added to the mixture under argon. The reaction mixture was heated under reflux for 3 days. Then, the solvent was evaporated under vacuum and cold $n$-hexane $(2 \mathrm{~mL})$ was added to the remaining content to form precipitate. The precipitate was filtered off and purified by column chromatography (silica gel 60/n-hexane : $\mathrm{CH}_{2} \mathrm{Cl}_{2}=1: 5$ ) to remove ruthenium complexes. Evaporation of the solvent gave an analytically pure sample (white or yellow powder).

By silylative coupling copolycondensation (SCC). For oligomers syntheses, complex ruthenium(II) $-\left[\operatorname{RuHCl}(\mathrm{CO})\left(\mathrm{PCy}_{3}\right)_{2}\right]$ was used as a catalyst. Solvent was dried and deoxygenated and all operations were performed under argon.

System 1. A mixture of silicon monomer-divinylsilsesquioxane $(\mathbf{1 A})\left(0.05 \mathrm{~g}, 4.13 \times 10^{-5} \mathrm{~mol}\right)$, distyrylarene $\left(4.13 \times 10^{-5}\right.$ $\mathrm{mol}),\left[\mathrm{RuHCl}(\mathrm{CO})\left(\mathrm{PCy}_{3}\right)_{2}\right]\left(0.0006 \mathrm{~g}, 8.26 \times 10^{-7} \mathrm{~mol}\right)$ and toluene $(1.5 \mathrm{~mL})$ was placed in a $5 \mathrm{~mL}$ two-necked glass reactor equipped with a magnetic stirring bar and condenser connected to a bubbler. The reaction mixture was heated at $110{ }^{\circ} \mathrm{C}$ and stirred for $72 \mathrm{~h}$. The reaction progress was monitored by ${ }^{1} \mathrm{H}$ NMR. After the coupling process was complete, the solvent was evaporated under vacuum and dichloromethane was added. Then, the final product was purified by repeated precipitation from methanol. The polymeric material was filtered off and dried under vacuum to give an analytically pure sample (white or yellow powder).

System 2. A mixture of silicon monomer-divinylsilsesquioxane (1A) $\left(0.05 \mathrm{~g}, 4.13 \times 10^{-5} \mathrm{~mol}\right)$, distyrylarene $\left(4.13 \times 10^{-5} \mathrm{~mol}\right)$, and dichloromethane $(2 \mathrm{~mL})$ was placed in a $5 \mathrm{~mL}$ two-necked glass reactor equipped with a magnetic stirring bar and condenser connected to a bubbler. The reaction mixture was heated up to $45^{\circ} \mathrm{C}$ and $\left[\mathrm{RuHCl}(\mathrm{CO})\left(\mathrm{PCy}_{3}\right)_{2}\right]\left(0.0006 \mathrm{~g}, 8.26 \times 10^{-7}\right.$ mol) was added. After 5 min copper(I) chloride $(0.0004 \mathrm{~g}, 4.13 \times$ $10^{-6} \mathrm{~mol}$ ) was added and the reaction mixture was heated under reflux for 3 days. The reaction progress was monitored by ${ }^{1} \mathrm{H}$ NMR. After the coupling process was complete, the solvent was evaporated under vacuum and cold $n$-hexane $(2 \mathrm{~mL})$ was added to the remaining content to form precipitate. The precipitate was filtered off and purified by column chromatography (silica gel 60/ $n$-hexane $\left.: \mathrm{CH}_{2} \mathrm{Cl}_{2}=1: 5\right)$ to remove ruthenium complexes. Evaporation of the solvent gave an analytically pure sample (white or yellow powder).

\section{Analytical data for isolated macromolecular compounds (9-15)}

9: $\quad \operatorname{poly}\{[9,19-(E)-4-(1-n a p h t h y l) s t y r y l m e t h y l]-1,3,5,7,11,13$, 15,17-octaphenylpentacyclo[11.7.1.1 $\left.1^{3,11} \cdot 1^{5,17} \cdot 1^{7,15}\right]$ decasiloxane $\}$ s. ${ }^{1} \mathrm{H}$ NMR (300 MHz, $\left.\mathrm{CDCl}_{3}, \delta, \mathrm{ppm}\right): 0.39$ (s, 3H, $\mathrm{SiCH}_{3}$ external), 0.49 (s, 3H, $\mathrm{SiCH}_{3}$, internal), 5.80-6.23 (dd, 3H, trace amount of signals coming from $\mathrm{Si}-H \mathrm{C}=\mathrm{CH}_{2}$ terminal), $6.62\left(\mathrm{~d}, 1 \mathrm{H}, J_{\mathrm{HH}}=\right.$ $18.3 \mathrm{~Hz},=\mathrm{CH}-\mathrm{Si}), 6.99-7.97(\mathrm{~m}, 56 \mathrm{H}$, aromatic parts and $=\mathrm{CH}-$ $\left.\mathrm{C}_{6} \mathrm{H}_{4}-\right) ;{ }^{13} \mathrm{C} \mathrm{NMR}\left(75 \mathrm{MHz}, \mathrm{CDCl}_{3}, \delta, \mathrm{ppm}\right): 0.11,0.14,124.45$, 126.04, 126.11, 126.31, 126.95, 127.45, 128.01, 130.36, 130.48, $132.02,132.14,134.16,134.35,136.81,138.05,139.74,146.32 ;{ }^{29} \mathrm{Si}$ NMR (79 MHz, $\left.\mathrm{CDCl}_{3}, \delta, \mathrm{ppm}\right):-30.19,-78.27,-79.51$.

10: $\operatorname{poly}\{[9,19-(E)-4-(1-n a p h t h y l) s t y r y l p h e n y l]-1,3,5,7,11,13$, 15,17-octaphenylpentacyclo[11.7.1.1 $\left.1^{3,11} \cdot 1^{5,17} \cdot 1^{7,15}\right]$ decasiloxane $\}$ s. ${ }^{1} \mathrm{H}$ NMR (300 MHz, $\left.\mathrm{CDCl}_{3}, \delta, \mathrm{ppm}\right): 6.69$ (d, $1 \mathrm{H}, J_{\mathrm{HH}}=18.7 \mathrm{~Hz}$, $=\mathrm{CH}-\mathrm{Si}), 6.98-8.27\left(\mathrm{~m}, 65 \mathrm{H},=\mathrm{CH}-\mathrm{C}_{6} \mathrm{H}_{4}-, \mathrm{C}_{6} \mathrm{H}_{5}\right.$ and naphthalene); ${ }^{13} \mathrm{C} \mathrm{NMR}\left(125 \mathrm{MHz}, \mathrm{CDCl}_{3}, \delta, \mathrm{ppm}\right): 122.71 ; 125.98,126.36$, $126.95,127.46,127.55,127.63,127.68,127.75,127.91$ (br s), 129.76, 130.23, 130.32, 130.52, 130.65, 130.81, 131.74, 131.86, $134.10,134.18,134.23,136.55,139.55,141.07,147.71,165.46 ;{ }^{29} \mathrm{Si}$ NMR (79 MHz, $\left.\mathrm{CDCl}_{3}, \delta, \mathrm{ppm}\right):-44.55,-77.90,-79.35$.

11: $\operatorname{poly}\{[9,19-(E)-4-(9-a n t h r a c e n y l) s t y r y l m e t h y l]-1,3,5,7,11$, 13,15,17-octaphenylpentacyclo $\quad\left[11.7 .1 .1^{3,11} \cdot 1^{5,17} \cdot 1^{7,15}\right]$ decasiloxane\}s. ${ }^{1} \mathrm{H}$ NMR $\left(300 \mathrm{MHz}, \mathrm{CDCl}_{3}, \delta, \mathrm{ppm}\right): 0.39\left(\mathrm{~s}, \mathrm{SiCH}_{3}\right.$ external), 0.49 (s, 3H, $\mathrm{SiCH}_{3}$, internal), 5.92-6.23 (dd, $3 \mathrm{H}$, trace amount of signals coming from $\mathrm{Si}-\mathrm{HC}=\mathrm{CH}_{2}$ terminal), 6.62 (d, $\left.1 \mathrm{H}, J_{\mathrm{HH}}=19.5 \mathrm{~Hz},=\mathrm{CH}-\mathrm{Si}\right), 6.91-7.81(\mathrm{~m}, 58 \mathrm{H}$, aromatic part and $\left.=\mathrm{CH}-\mathrm{C}_{6} \mathrm{H}_{4}-\right) ;{ }^{13} \mathrm{C} \mathrm{NMR}\left(75 \mathrm{MHz}, \mathrm{CDCl}_{3}, \delta, \mathrm{ppm}\right):-0.71$, 125.25, 126.89, 127.07, 129.95, 130.61, 131.61, 134.17, 134.33, 136.98, 147.90; ${ }^{29} \mathrm{Si} \mathrm{NMR}\left(79 \mathrm{MHz}, \mathrm{CDCl}_{3}, \delta, \mathrm{ppm}\right):-30.46$, $-78.27,-78.36,-79.51$.

12: $\operatorname{poly}\{[9,19-(E)-4-(9-a n t h r a c e n y l) s t y r y l p h e n y l-1,3,5,7,11$, $13,15,17-o c t a p h e n y l p e n t a c y c l o \quad\left[11.7 .1 .1^{3,11} \cdot 1^{5,17} \cdot 1^{7,15}\right]$ decasiloxane\}s. ${ }^{1} \mathrm{H}$ NMR (400 MHz, $\left.\mathrm{CD}_{2} \mathrm{Cl}_{2}, \delta, \mathrm{ppm}\right): 6.86(\mathrm{~d}, 1 \mathrm{H}$, $\left.J_{\mathrm{HH}}=19.2 \mathrm{~Hz},=\mathrm{CH}-\mathrm{Si}\right), 7.03-8.74\left(\mathrm{~m}, 68 \mathrm{H},=\mathrm{CH}-\mathrm{C}_{6} \mathrm{H}_{4}-\right.$ and aromatic parts); ${ }^{13} \mathrm{C} \mathrm{NMR}$ (125 MHz, $\mathrm{CD}_{2} \mathrm{Cl}_{2}, \delta$, ppm): 123.36, $125.15,126.83$ (t, $J=6.1 \mathrm{~Hz}), 127.56,127.60,127.65,127.71$, $127.77,127.94,127.99,128.47,129.61,130.42$, 130.47, 130.71, 131.51, 133.19, 134.00, 134.05, 134.11, 134.16, 137.38, 140.12, 147.53, 165.38; ${ }^{29} \mathrm{Si} \mathrm{NMR}\left(79 \mathrm{MHz}, \mathrm{CD}_{2} \mathrm{Cl}_{2}, \delta, \mathrm{ppm}\right):-44.63$, $-77.77,-79.25$.

13: poly $\{[9,19-(E)-4-(2,3,5,6-$ tetrafluorophenyl)styrylmethyl $]$ $1,3,5,7,11,13,15,17-o c t a p h e n y l p e n t a c y c l o\left[11.7 .1 .1^{3,11} \cdot 1^{5,17} \cdot 1^{7,15}\right]$ decasiloxane $\}$ s. ${ }^{1} \mathrm{H}$ NMR (300 $\left.\mathrm{MHz}, \mathrm{CDCl}_{3}, \delta, \mathrm{ppm}\right): 0.39$ (s, $\mathrm{SiCH}_{3}$ external), 0.48 (s, 3H, $\mathrm{SiCH}_{3}$, internal), 5.35 (d, $1 \mathrm{H}, J_{\mathrm{HH}}=$ $10.5 \mathrm{~Hz}$, trace amount of signals coming from $>\mathrm{C}-\mathrm{HC}=\mathrm{CH}_{2}$ ), $5.84\left(\mathrm{~d}, 1 \mathrm{H}, J_{\mathrm{HH}}=17.1 \mathrm{~Hz}\right.$, trace amount of signals coming from ${ }_{\mathrm{C}-\mathrm{HC}}=\mathrm{CH}_{2}$ ), $6.55\left(\mathrm{~d}, 1 \mathrm{H}, J_{\mathrm{HH}}=19.2 \mathrm{~Hz},=\mathrm{CH}-\mathrm{Si}\right), 6.79(\mathrm{dd}$, $1 \mathrm{H}$, trace amount of signals coming from $\left.>\mathrm{C}-\mathrm{HC}=\mathrm{CH}_{2}\right), 6.88-$ $7.76\left(\mathrm{~m}, 49 \mathrm{H}\right.$, aromatic parts and $1 \mathrm{H}$ coming from $\left.=\mathrm{CH}-\mathrm{C}_{6} \mathrm{H}_{4}-\right)$; ${ }^{13} \mathrm{C}$ NMR (75 MHz, $\left.\mathrm{CDCl}_{3}, \delta, \mathrm{ppm}\right):-1.13,-0.87,125.95,126.55$, $127.11,127.41,127.76,127.93,128.01,130.43,130.51,130.88$, 132.04, 134.12, 134.26, 145.75; ${ }^{29} \mathrm{Si} \mathrm{NMR}\left(79 \mathrm{MHz}, \mathrm{CDCl}_{3}, \delta\right.$, ppm): $-30.50,-78.20,-78.24,-78.95,-79.50$.

14: $\operatorname{poly}\{[9,19-(E)-4-(2,3,5,6$-tetrafluorophenyl)styrylphenyl]$1,3,5,7,11,13,15,17-o c t a p h e n y l p e n t a c y c l o\left[11.7 .1 .11^{3,11} \cdot 1^{5,17} \cdot 1^{7,15}\right]$ decasiloxane $\}$ s. ${ }^{1} \mathrm{H} \mathrm{NMR}\left(400 \mathrm{MHz}, \mathrm{CD}_{2} \mathrm{Cl}_{2}, \delta, \mathrm{ppm}\right): 6.75(\mathrm{~d}, 1 \mathrm{H}$, $\left.J_{\mathrm{HH}}=19.2 \mathrm{~Hz},=\mathrm{CH}-\mathrm{Si}\right), 6.98-8.27\left(\mathrm{~m}, 67 \mathrm{H},=\mathrm{CH}-\mathrm{C}_{6} \mathrm{H}_{4}-\right.$ and aromatic parts); ${ }^{13} \mathrm{C}$ NMR (125 $\left.\mathrm{MHz}, \mathrm{CD}_{2} \mathrm{Cl}_{2}, \delta, \mathrm{ppm}\right): 123.93$, 127.02, 127.61 (t, $J=8.6 \mathrm{~Hz}), 127.96,130.30,130.34,130.37$, 130.41, 130.49, 130.56, 130.67 (t, $J=3.7 \mathrm{~Hz}), 131.46,134.00$ (d, $J=7.9 \mathrm{~Hz}), 138.19,147.21 ;{ }^{29} \mathrm{Si} \mathrm{NMR}\left(79 \mathrm{MHz}, \mathrm{CD}_{2} \mathrm{Cl}_{2}, \delta, \mathrm{ppm}\right)$ : $-44.71,-77.81,-79.31$. 
15: $\quad \operatorname{poly}\{[9,19-(E)-4-(2-t h i e n y l) s t y r y l m e t h y l]-1,3,5,7,11,13$, 15,17-octaphenylpentacyclo[11.7.1.1 $\left.{ }^{3,11} \cdot 1^{5,17} \cdot 1^{7,15}\right]$ decasiloxane $\} \mathrm{s}$. ${ }^{1} \mathrm{H}$ NMR (300 MHz, $\left.\mathrm{CDCl}_{3}, \delta, \mathrm{ppm}\right): 0.38$ (s, $\mathrm{SiCH}_{3}$ external), 0.46 (s, $3 \mathrm{H}, \mathrm{SiCH}_{3}$, internal), 5.86-6.25 (dd, $3 \mathrm{H}$, trace amount of signals coming from $\mathrm{Si}-\mathrm{HC}=\mathrm{CH}_{2}$ terminal), $6.46\left(\mathrm{~d}, 1 \mathrm{H}, J_{\mathrm{H}-\mathrm{H}}=19.2 \mathrm{~Hz}\right.$, $=\mathrm{CH}-\mathrm{Si}), 6.85-7.80(\mathrm{~m}, 52 \mathrm{H}$, aromatic part and $-\mathrm{Si}-\mathrm{HC}=\mathrm{CH}-)$; ${ }^{13} \mathrm{C} \mathrm{NMR}\left(125 \mathrm{MHz}, \mathrm{CDCl}_{3}, \delta, \mathrm{ppm}\right):-1.14,-0.70,124.21,125.32$, $125.53,127.41,127.62,127.85,128.24,129.05,130.42,131.96$, 134.01, 134.12, 134.22, 137.89, 145.89; ${ }^{29} \mathrm{Si} \mathrm{NMR} \mathrm{(79} \mathrm{MHz,} \mathrm{CDCl}_{3}$, $\delta, \mathrm{ppm}):-30.15,-78.26,-79.41,-79.51$.

\section{Results and discussion}

Taking into regard to the positive results presented in our recent paper on the possibility of using cross-metathesis reaction and silylative coupling in functionalization of divinylsubstituted double-decker silsesquioxanes, we decided to expand the palette of tested olefins to the compounds containing conjugated double bond system. The premise for this is still undiminished interest in silsesquioxanes derivatives, their limited commercial availability and consequently high prices. Therefore, it seems reasonable to perform studies aimed at optimizing and modifying this group of organosilicon compounds.

In studies presented in this paper, we focused on the functionalization of two types of double-decker silsesquioxane derivatives containing methyl (1A) or phenyl (1B) substituents located at 9 and 19 silicon atom of the core. Both substrates were prepared by a corner-capping reaction of incompletely condensed silsesquioxane DDSQ-4OH with vinyldichloro(organo)silane. This synthetic procedure was thoroughly described in our last paper. ${ }^{11 b}$ In view of the fact that methyl-substituted silicon derivatives are not reactive in metathetic transformation, DDSQ-2Sivi (1B) bearing phenyl substituents were applied to functionalization based on crossmetathesis reaction, while DDSQ-2Sivi (1A) with methyl groups was tested in silylative coupling reaction (Scheme 3).
Cross-metathesis of phenyl-substituted divinylsilsesquioxane (1B) with 1-(4-vinylphenyl)naphthalene was chosen as a model reaction for optimization studies of silsesquioxane containing two vinyl groups. Treatment of divinysilsesquioxane (1B) with two equivalents of 1-(4-vinylphenyl)naphthalene in the presence of Grubbs catalyst $\left[\mathrm{RuCl}_{2}\left(\mathrm{PCy}_{3}\right)_{2}(=\mathrm{CHPh})\right](1 \% \mathrm{~mol}$ in relation to vinyl group of DDSQ-2SiVi (1B) in refluxing dichloromethane, resulted in its efficient transformation. Due to the large molecular mass of the silsesquioxyl substrate (1B), the reaction progress could not be monitored by GC. After $24 \mathrm{~h}$, the reaction mixture was subjected to the ${ }^{1} \mathrm{H}$ NMR analysis which indicated nearly complete conversion of both substrates revealing selective formation of expected product (2). Similar reaction completion was observed when other 4-styryl substituted derivatives and 1B were used as reaction partners. The results obtained were collected in Table 1 . In each case, the reaction led to efficient and selective formation of $E$-isomer of the desired product. All obtained products (1-4) are air-stable,

Table 1 Cross-metathesis of divinyl-substituted silsesquioxanes (1B) with 4-styryl substituted derivatives ${ }^{a}$

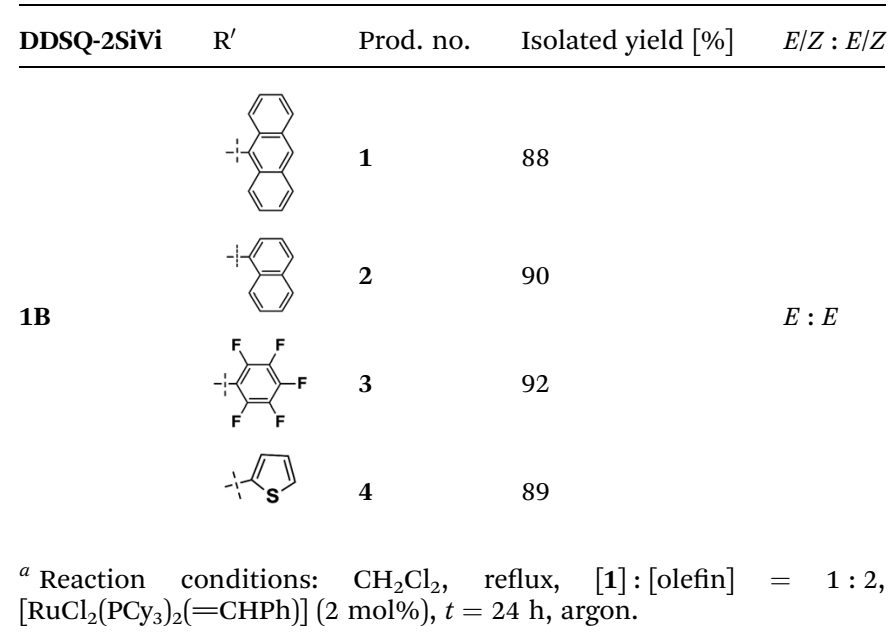
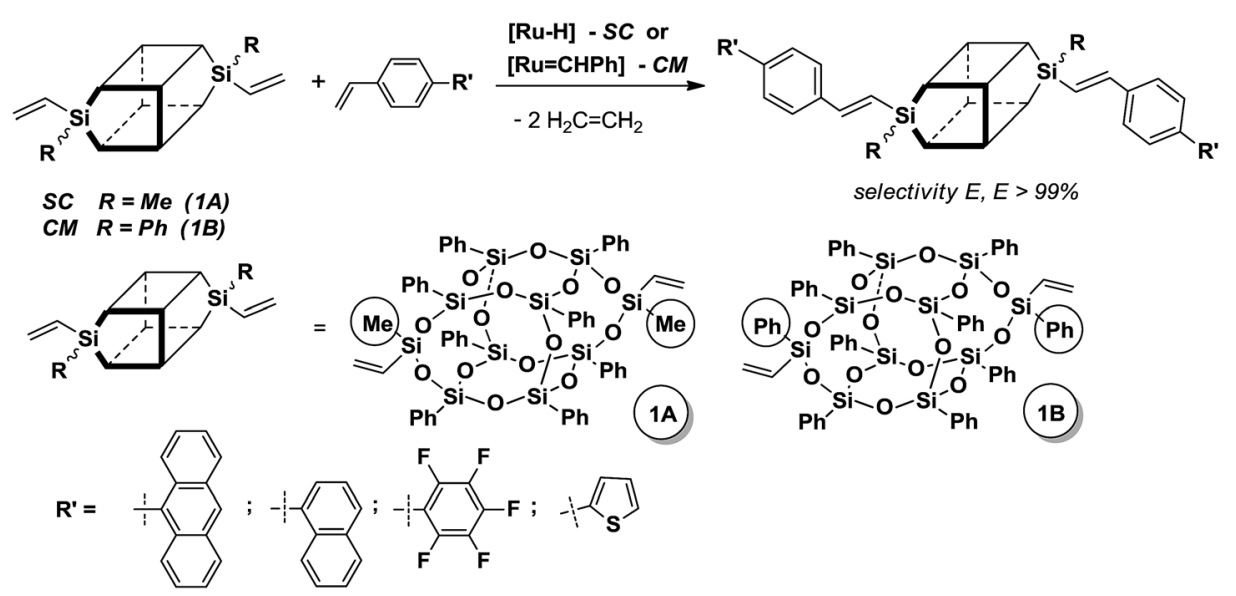

Scheme 3 Silylative coupling and cross-metathesis of divinyl-substituted double-decker silsesquioxanes (1A, 1B) with 4-styryl substituted derivatives. 
Table 2 Silylative coupling of divinyl-substituted silsesquioxanes (1A) with 4-styryl substituted derivatives ${ }^{a}$

\begin{tabular}{|c|c|c|c|}
\hline & & & Isolated \\
\hline DDSQ-2Si & $\mathrm{R}^{\prime}$ & Prod. no. & System 1 \\
\hline
\end{tabular}

$1 \mathrm{~A}$

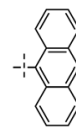

5

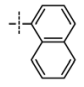

6

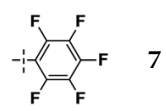

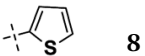

72

81

86

$62 *$
85

90

91

89
${ }^{a}$ Reaction conditions: system 1 : toluene, $90{ }^{\circ} \mathrm{C},[\mathbf{1}]:[$ olefin] $=1: 3$, $\left[\mathrm{RuHCl}(\mathrm{CO})\left(\mathrm{PCy}_{3}\right)_{2}\right](2 \mathrm{~mol} \%), t=24 \mathrm{~h}$, argon; $* 110{ }^{\circ} \mathrm{C}, t=96 \mathrm{~h}$. System 2: $\mathrm{CH}_{2} \mathrm{Cl}_{2}, 45^{\circ},[1]:\left[\right.$ olefin] $=1: 2,\left[\operatorname{RuHCl}(\mathrm{CO})\left(\mathrm{PCy}_{3}\right)_{2}\right](2$ $\operatorname{mol} \%),[\mathrm{Ru}]:[\mathrm{CuCl}]=1: 5, t=24 \mathrm{~h}$, argon.

easy to handle white or yellow solids and can be purified by column chromatography.

In the next experiments, silylative coupling of methylsubstituted divinylsilsesquioxane (1A) with 4-styryl substituted derivatives was performed. A series of catalytic tests aimed at optimizing the reaction systems for efficient and selective synthesis of dialkenyl-substituted derivatives (5-8) was carried out. Optimization involved selection of a suitable solvent, ratios of reactants, concentration of catalyst used, reaction temperature and time as well as determination of possible influence of cocatalyst on the reaction course. According to obtained results of performed tests, effective functionalization of $\mathbf{1 A}$ based on silylative coupling may be conducted in two ways concerning different reaction conditions. The first reaction was carried out in toluene at $90{ }^{\circ} \mathrm{C}$ or $110^{\circ} \mathrm{C}$ under constant flow of argon in the presence of ruthenium(II) hydride complex $\left[\mathrm{RuHCl}(\mathrm{CO})\left(\mathrm{PCy}_{3}\right)_{2}\right]$ used at $2 \mathrm{~mol} \%$ ( $1 \% \mathrm{~mol}$ in relation to vinyl group of DDSQ2SiVi (1A). Similar effect can be achieved by introducing a cocatalyst to the reaction system which is copper(I) chloride. The addition of the afore-mentioned salt helps to reduce the reaction temperature to $45{ }^{\circ} \mathrm{C}$ and change the solvent from toluene to methylene chloride. The results obtained are collected in Table 2.

The mechanism explaining the effect of copper(I) chloride addition on the reaction course was not examined. A reasonable explanation reported in literature for similar systems indicates that copper salt acts as a phosphine scavenger and thus facilitates the formation of catalytically active species. ${ }^{14,15}$ Both reaction systems were tested using different 4 -styryl substituted derivatives and in each case complete conversion of silsesquioxyl substrate $\mathbf{1 A}$ was achieved.

The results presented in Table 2 show that irrespective of the type of olefin used, all reactions are stereoselective and lead exclusively to the product of $E$ geometry of the resulting substituted vinylene bond $\left({ }^{1} \mathrm{H}\right.$ NMR data along with the high values of $J_{\mathrm{HH}}$ coupling constant are to confirm this). The reactions performed with copper chloride(I) allow the preparation of the desired products under mild reaction conditions. However, it is necessary to isolate the 4-styryl substituted derivatives of $\mathbf{1 A}$ from the reaction mixtures on chromatographic columns. This necessity follows mainly from poor solubility of the copper salt used in the solvent that precipitates silsesquioxyl products (methanol, acetone, $n$-hexane). Furthermore, these systems may be applied only in the case of olefins soluble in refluxing methylene chloride. Applying harsh conditions, i.e. toluene, $90{ }^{\circ} \mathrm{C}$ or $110^{\circ} \mathrm{C}$, promotes (or increases) the homogeneity of the system as with increasing temperature the solubility of most styryl derivatives also increases. Therefore, these systems can be advantageously used for substrates that cause solubility problems. Both developed reaction systems are characterized by high stereoselectivity and selection of appropriate conditions for functionalization largely depend on the type olefins used.

\section{Synthesis of macromolecular compounds with DDSQ embedded in the linear chain - ADMET and silylative coupling copolycondensation}

Positive results obtained for the functionalization of divinylsilsesquioxanes via cross-metathesis and silylative coupling with
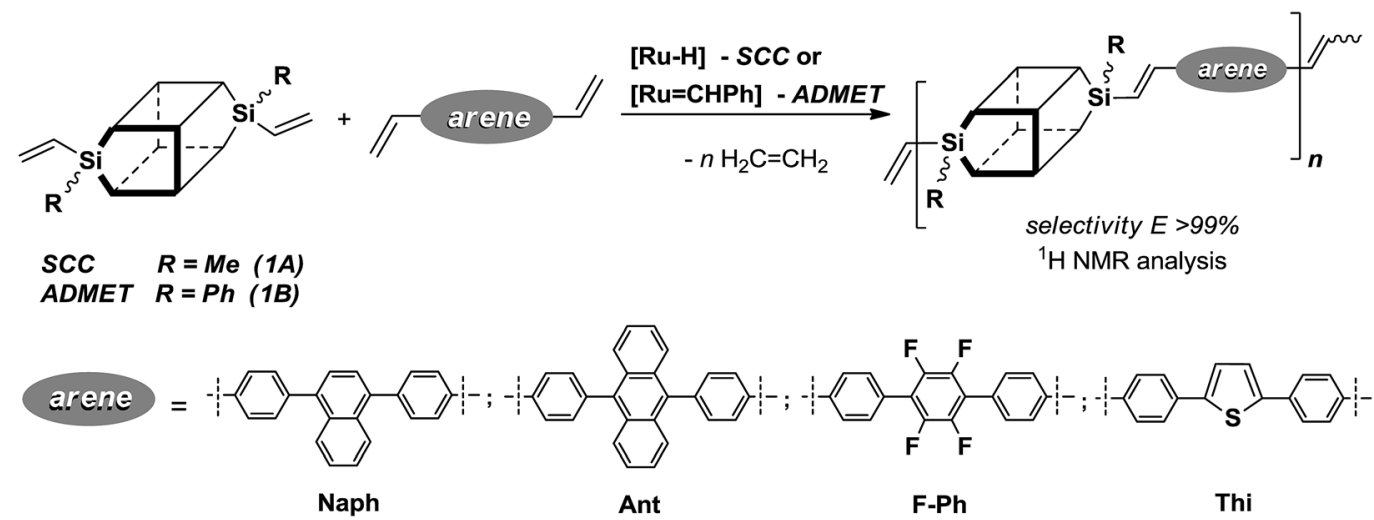

Scheme 4 Silylative coupling copolycondensation (SCC) and metathetic copolymerisation (ADMET) of divinyl-substituted double-decker silsesquioxanes $(1 \mathrm{~A}, 1 \mathrm{~B})$ with diolefins. 
Table 3 Silylative coupling copolycondensation and ADMET of divinyl-substituted silsesquioxanes DDSQ-2SiVi (1A, 1B) with diolefins ${ }^{a}$

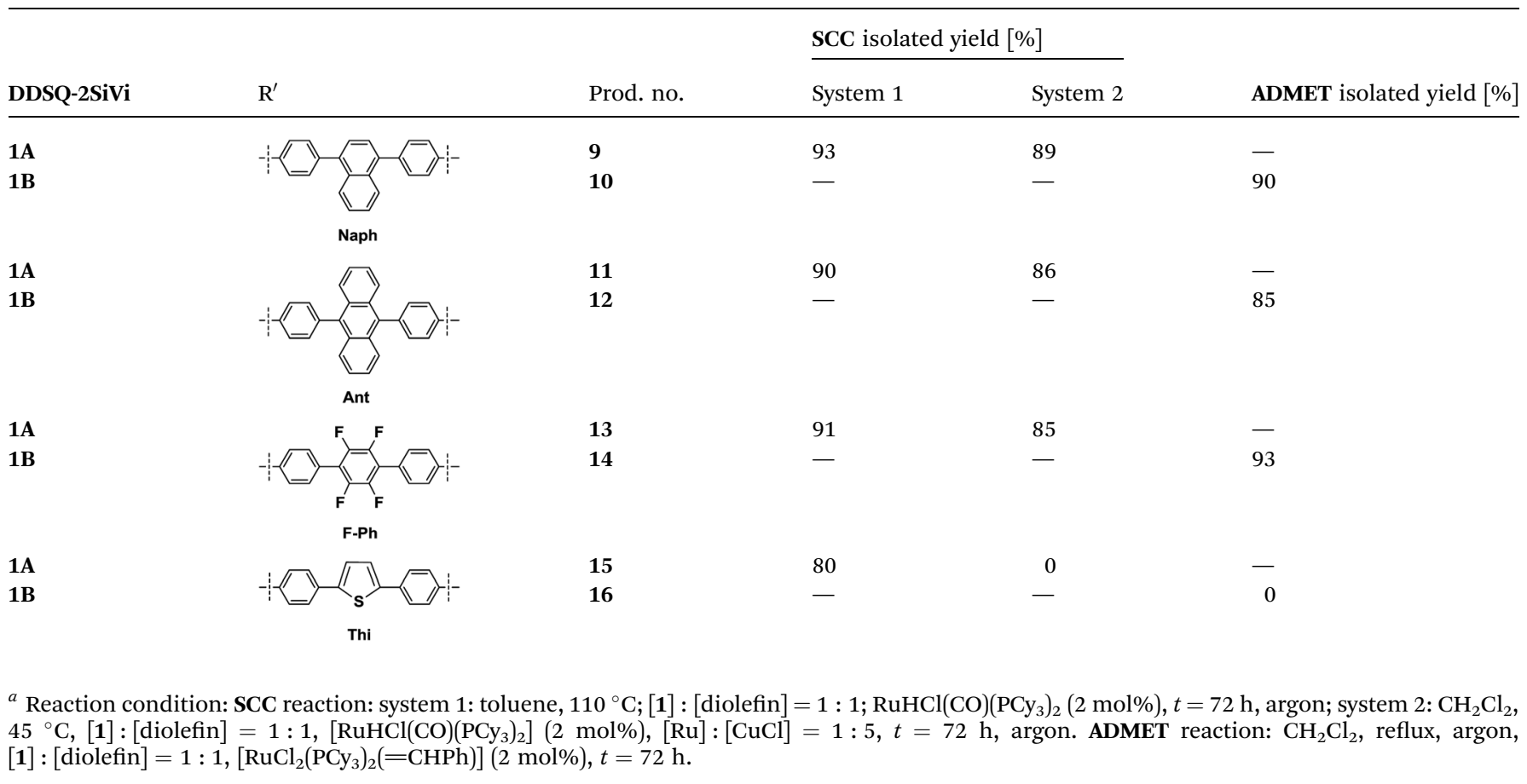

a series of para-substituted styrenes with highly $\pi$-conjugated substituents prompted us to extend the range of olefins to diolefins. According to literature processes, we synthesized a number of distyrylarenes, which were then subjected to SCC and ADMET copolymerization with DDSQ-2SiVi. The overview of this processes is presented in Scheme 4.

As a result of a series of catalytic tests, successful procedures that ensure the efficiency of both reactions were developed. They allow avoidance of generation of the undesirable polyarenes without the need for any inhibitor addition. Analogously, as in the synthesis of the molecular products, for the studies on metathetic copolymerization (ADMET), phenyl-substituted silsesquioxane (1B) was applied exclusively whereas in the silylative coupling copolycondensation double-decker silsesquioxane derivative with methyl group at 9 and 19 silicon atom (1A) was used. After optimization of the reaction conditions, a series of copolymers of stereoregular DDSQ-silylene-vinylene-arylene units was obtained (Table 3). As indicated in Table 3, metathetic transformation failed to give cooligomer containing sulfur atom in its structure (16). This may be associated with the

Table 4 Results of GPC analysis of oligomers obtained

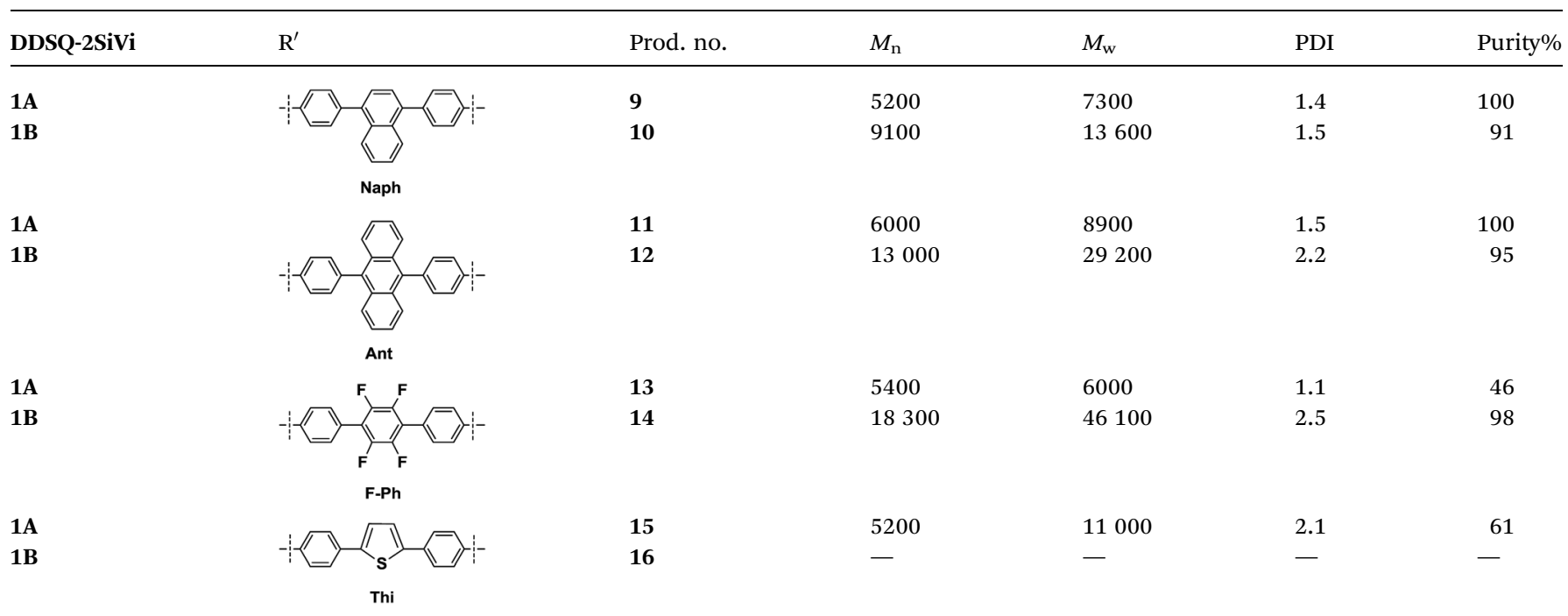




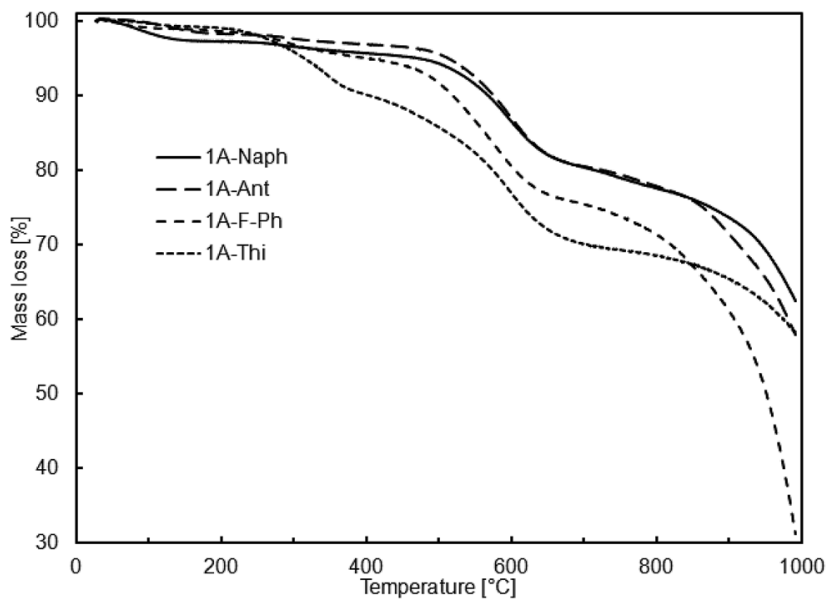

Fig. 1 TGA curves of obtained cooligomers via SCC.

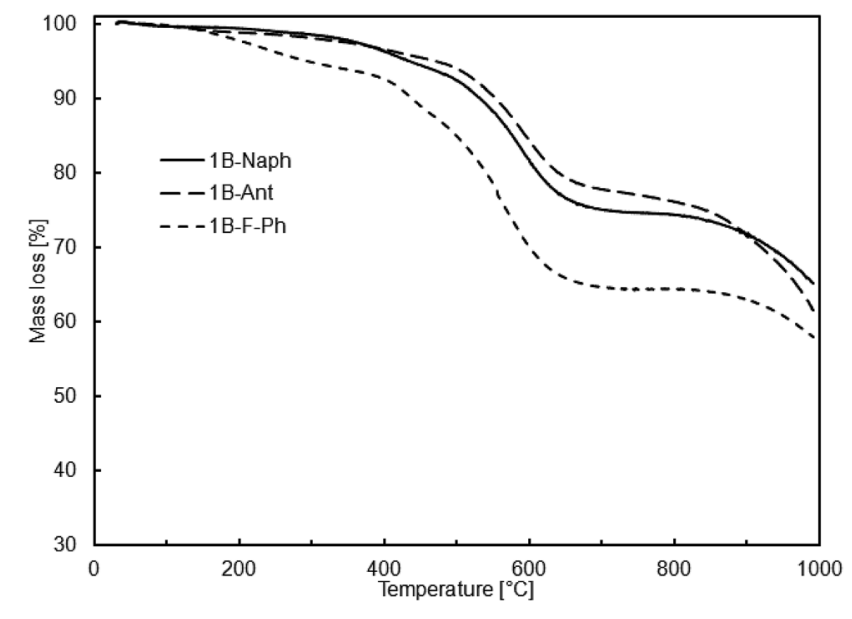

Fig. 2 TGA curves of obtained cooligomers via ADMET copolymerization. lack of solubility of the distyrylthiophene (Thi) used in refluxing methylene chloride. There was also no reaction progress observed when increasing the amount of the solvent used or when twofold load of alkylidene catalyst was used. To the best of our knowledge, no experiments with Grubbs I generation catalyst have been conducted at high temperatures because of its thermal degradation and in situ formation of ruthenium hydride species that catalyze silylative coupling process. ${ }^{16} \mathrm{Co}-$ oligomer containing thiophene derivative was synthesized only by SCC in the high temperature system ensuring the solubility of the diolefin used.

The new oligomeric products obtained were purified by twice repeated precipitation in methanol or $n$-hexane. Products obtained in the presence of catalytic systems containing copper chloride(I) required further purification on column chromatography with silica gel because the co-catalyst also was not soluble in the precipitant solvent. All oligomeric materials are white or yellow solids and show good solubility in common organic solvents, e.g. THF, $\mathrm{CHCl}_{3}$ and $\mathrm{CH}_{2} \mathrm{Cl}_{2}$. They were analyzed by spectroscopic methods.

Subsequently, all prepared samples were analyzed by gel permeation chromatography. They were also subjected to thermogravimetric analysis to evaluate the effect of the comonomers used and the type of catalytic process on their molecular weights, molecular weight distribution and thermal stability. GPC results obtained for cooligomers synthesized via silylative coupling copolycondensation (SCC) and ADMET copolymerization processes are summarized in Table 4 .

On the basis of the data obtained, it can be noted that ADMET process led to formation of products of relatively higher molecular weights $\left(M_{\mathrm{n}}\right.$ and $\left.M_{\mathrm{w}}\right)$ and higher PDI values except for co-oligomer containing thiophene derivative. These results are in good agreement with those in the few available papers concerning the synthesis of similar linear polymers with a DDSQ

Table 5 Thermal properties of obtained cooligomers

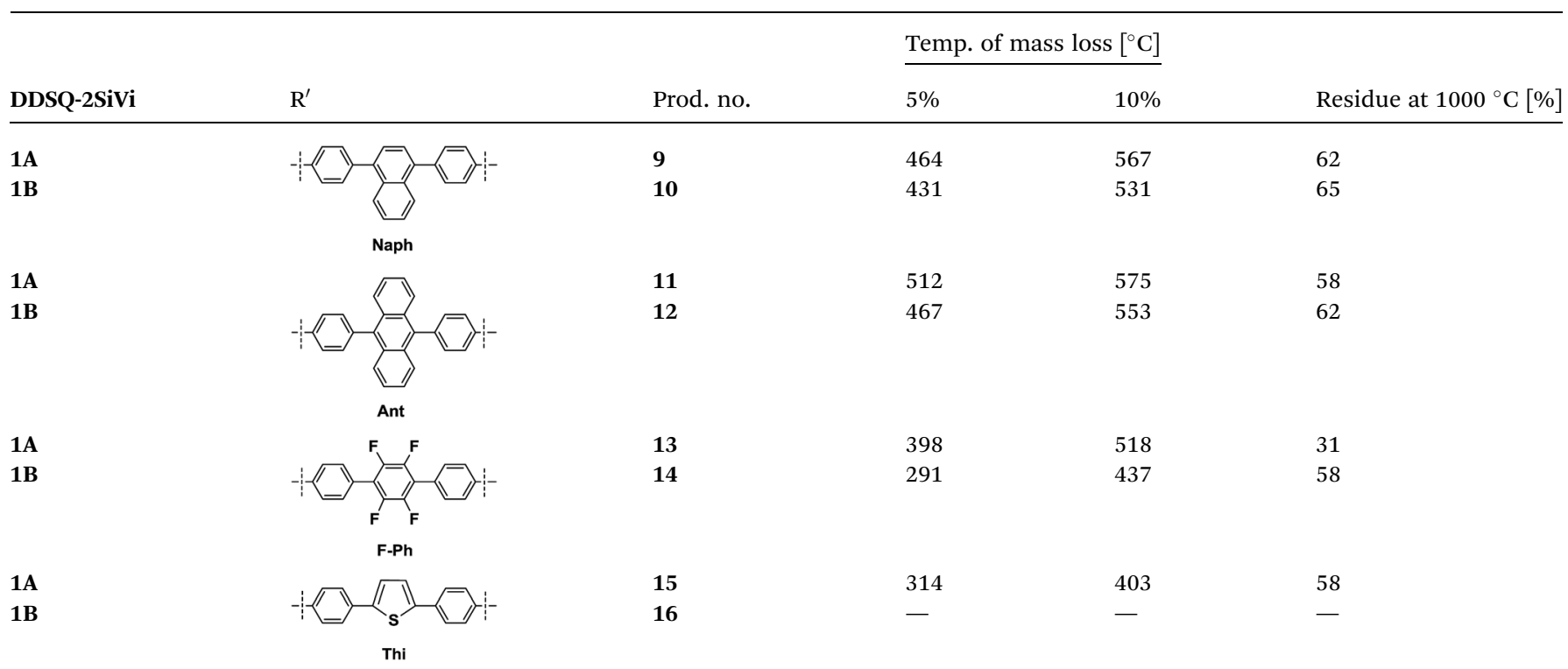


spacer and $\pi$-conjugated arenes. Kakimoto et al. obtained analogous systems with $M_{\mathrm{n}}$ weight in the range of 11900 to 29100 , and $M_{\mathrm{w}} / M_{\mathrm{n}}$ values of $2.9-4.9 .^{3 e}$

According to the thermogravimetric studies, all prepared cooligomers show relatively high thermal stability (Fig. 1 and 2).

It is worth noting that there is relatively good correlation between the sample thermal stability and its purity or more precisely, the content of higher molecular weight fraction. It may be an explanation why the samples obtained via SCC process are more thermally stable despite their relatively lower molecular weights in comparison to the samples obtained via ADMET copolymerization (Tables 4 and 5).

So far, there has been scarce information on similar oligomeric systems with DDSQ fragments. However, among those rare examples in literature, high temperatures of $10 \%$ mass loss, reaching over $550{ }^{\circ} \mathrm{C}$, are characteristic of DDSQ copolymers irrespective of the comonomer or catalytic process used. ${ }^{3 h}$ This effect may be also attributed to the general tendency of the high thermal stability of POSS compounds heaving phenyl groups. $^{3 e}$

\section{Conclusions}

Recent years have shown that the concept of synthesis of molecular and macromolecular organosilicon compounds based on DDSQ led to two major trends in their development. Molecular derivatives based on a DDSQ core are a class of compounds that may be synthesized using, among others, stoichiometric types of modifications, i.e. a condensation of tetrasilanolphenyl POSS (DDSQ-4OH) with alkoxy- or chlorosilanes. On the other hand, difunctional DDSQ compounds with reactive groups, e.g. a $\mathrm{Si}-\mathrm{H}$ or $\mathrm{Si}-\mathrm{HC}=\mathrm{CH}_{2}$ bond, are ideal substrates for further catalytic modifications. We described divinyl-substituted double-decker silsesquioxanes that undergo efficient functionalization via cross-metathesis and silylative coupling reactions with a series of para-substituted styrenes that possess highly $\pi$-conjugated substituents. For all styrene derivatives tested, both reactions proceeded effectively and with high stereoselectivity leading to exclusive formation of $E$ isomer. A divinyl-substituted double-decker silsesquioxane containing a methyl group located at Si-9 and Si-19 atoms (1A) was inactive in metathetic transformation, but this result was expected in the context of our earlier reports. ${ }^{17}$ Cross-metathesis and silylative coupling silylative are convenient and complementary synthetic routes leading to functionalization of vinylsubstituted silicon derivatives. However, the choice of the optimum catalytic method depends on the specific character of the reaction system. The other synthetic path for DDSQ applications is to try to embed them into the main chain of the polymeric system. We proved that both, SCC and ADMET reactions may be used for the stereoselective synthesis of a new class of vinylene-arylene copolymers containing DDSQ in the main chain. New copolymeric material was characterized in terms of molecular weights, PDI as well as thermal analysis. TG analysis has proved the high level of thermal resistance of the obtained systems, reaching over $550{ }^{\circ} \mathrm{C}$, that is in close correlation with DDSQ copolymers.

\section{Acknowledgements}

The authors gratefully acknowledge the financial support from the National Science Centre (Poland) (MAESTRO Project No. DEC-2011/02/A/ST5/00472, SONATA Project No. DEC-2012/05/D/ ST5/03348).

\section{Notes and references}

1 (a) D. B. Cordes, P. D. Lickiss and F. Rataboul, Chem. Rev., 2010, 110, 2081; (b) D. B. Cordes and P. D. Lickiss, in Applications of Polyhedral Oligomeric Silsesquioxanes, Advances in Silicon Science 3, ed. C. Hartmann-Thompson, Springer, 2011.

2 (a) Y. Morimoto, K. Watanabe, N. Ootake, J. Inagaki, K. Yoshida and K. Ohguma, EP 1428795 (A1), 2004; US 7 169873 (B2), 2007; US 7449539 (B2), 2008; (b) K. Yoshida, Polymer Preprints Japan, 2003, 52, 316; (c) N. Ootake and K. Yoshida, US pat., 2006/0155091(A1), 2006.

3 (a) A. C. Kucuk, J. Matsui and T. Miyashita, Thin Solid Films, 2013, 534, 577; (b) B. Seurer, V. Vij, T. Haddad, J. M. Mabry and A. Lee, Macromolecules, 2010, 43, 9337; (c) M. Kohri, J. Matsui, A. Watanabe and T. Miyashita, Chem. Lett., 2010, 39, 1162; (d) J. Espinas, J. D. A. Pelletier, E. Abou-Hamad, L. Emsley and J.-M. A. Basset, Organometallics, 2012, 31, 7610; (e) M. Seino, T. Hayakawa, Y. Ishida, M. Kakimoto, K. Watanabe and H. Oikawa, Macromolecules, 2006, 39, 3473; (f) K. Yoshida, T. Hattori, N. Ootake, R. Tanaka and H. Matsumoto, Silsesqioxane-based polymers: synthesis of phenyl silsesquioxanes with double-decker structure and their polymers, in Silicon Based Polymers. Advances in Synthesis and Supramolecular Organization, ed. F. Ganachaud, S. Boileau and B. Boury, Springer, LondonNew York, 2008, p. 205; (g) S. Wu, T. Hayakawa, M. Kakimoto and H. Oikawa, Macromolecules, 2008, 41, 3481; (h) M. Miyasaka, Y. Fujiwara, H. Kudo and T. Nishikubo, Polym. J., 2010, 42, 799; ( $i$ ) K. Wei, L. Wang and S. Zheng, Polym. Chem., 2013, 4, 1491; (j) K. Wei, L. Wang and S. Zheng, J. Polym. Sci., Part A: Polym. Chem., 2013, 51, 4221; (k) W. Zhang, J. Xu, X. Li, G. Song and J. Mu, J. Polym. Sci., Part A: Polym. Chem., 2014, 52, 780; $(l)$ K. Wei, L. Wang, L. Li and S. Zheng, Polym. Chem., 2015, 6, 256; $(m)$ B. Dudziec and B. Marciniec, Curr. Org. Chem., 2015, DOI: 10.2174/1385272820666151228193728.

4 (a) B. Marciniec, Coord. Chem. Rev., 2005, 249, 2374; (b) B. Marciniec, Acc. Chem. Res., 2007, 40, 943; (c) B. Marciniec and C. Pietraszuk, in Green Metathesis Chemistry. Great Challenges in Synthesis Catalysis and Nanotechnology, ed. V. Dragutan, A. Demonceau, I. Dragutan and E. S. Finkelshtein, Springer, Berlin, 2010, p. 157; (d) C. Pietraszuk, P. Pawluć and B. Marciniec, in Handbook on Metathesis, R. Grubbs and D. J. O'Leary, 2015, vol. 2, 9, pp. 583-631.

5 (a) Y. Itami, B. Marciniec and M. Kubicki, Chem.-Eur. J., 2004, 10, 1239; (b) J. Waehner, B. Marciniec and P. Pawluc, Eur. J. Inorg. Chem., 2007, 2975; (c) P. Żak, C. Pietraszuk, B. Marciniec, G. Spolnik and W. Danikiewicz, Adv. Synth. 
Catal., 2009, 351, 2675; (d) P. Żak, B. Marciniec, M. Majchrzak and C. Pietraszuk, J. Organomet. Chem., 2011, 696, 887.

6 F. J. Feher, D. Soulivong, A. G. Eklund and K. D. Wydham, Chem. Commun., 1997, 1185.

7 S. Sulaiman, A. Bhaskar, J. Zhang, R. Guda, T. Goodson III and R. M. Laine, Chem. Mater., 2008, 20, 5563.

8 (a) G. Cheng, N. R. Vautravers, R. E. Morris and D. J. ColeHamilton, Org. Biomol. Chem., 2008, 6, 4662; (b) N. R. Vautravers, P. Andre, A. M. Z. Slavin and D. J. ColeHamilton, Org. Biomol. Chem., 2009, 7, 717; (c) N. R. Vautravers, P. Andre and D. J. Cole-Hamilton, J. Mater. Chem., 2009, 19, 4545.

9 H. Araki and K. Naka, J. Polym. Sci., Part A: Polym. Chem., 2012, 50, 4170.

10 M. Lo Conte, S. Staderini, A. Chambery, N. Berthet, P. Dumy, O. Renaudet, A. Marra and A. Dondoni, Org. Biomol. Chem., 2012, 10, 3269.
11 (a) P. Żak, B. Dudziec and B. Marciniec, US Appl. US 14,640,618, EP 15461513.2, 2015; (b) P. Żak, B. Dudziec, M. Kubicki and B. Marciniec, Chem.-Eur. J., 2014, 20, 9387.

12 (a) M. Majchrzak, S. Kostera, M. Kubicki and I. Kownacki, Dalton Trans., 2013, 42, 15535; (b) M. Majchrzak, M. Hybsz, S. Kostera, M. Kubicki and B. Marciniec, Tetrahedron Lett., 2014, 55, 3055.

13 C. S. Yi, D. W. Lee and Y. Chen, Organometallics, 1999, 18, 2043.

14 P. Żak, M. Kubicki, B. Marciniec, S. Rogalski, C. Pietraszuk and D. Frąckowiak, Dalton Trans., 2014, 43, 7911.

15 E. L. Dias, S. T. Nguyen and R. H. Grubbs, J. Am. Chem. Soc., 1997, 119, 3887.

16 B. Schmidt, Eur. J. Org. Chem., 2004, 1865.

17 C. Pietraszuk and H. Fischer, Chem. Commun., 2000, 2463. 